$\xi=-1$

\title{
A Faith Organization Scheme Model for Cloud
}

\author{
A. Vijaya Kumar ${ }^{1}$, K. Hemanth Kumar ${ }^{2}$ J. Tajudeen ${ }^{3}$, C. Suresh ${ }^{4}$ \\ ${ }^{I}$ Asst Professor Dept of CSE, KL E F, Vaddeswaram, India \\ ${ }^{2,3,4}$ Dept of CSE, KL E F, Vaddeswaram, India \\ *Corresponding author E-mail: hemanthkommineni6@gmail.com
}

\begin{abstract}
IT Industries are rapidly migrating their businesses to the cloud architecture. The exponential change is because of elasticity and low-cost services offered by the cloud. The High-performance services offered at low price attract customers to run their businesses on cloud. Other side of the coin Faith on data privacy offered by the cloud is a key issue for every customer. Although the CSP ensure about the privacy and security of the user's data, but the customer reliability on cloud is still low. This paper briefed the necessity of faith organization scheme, it overviewed various recent works done in this area. The comparative analysis enables us the scope to focus on the lacking of Faith organization on cloud. This paper focuses on various existing techniques "provable data possession" and "evidences of hopelessness" which are used to control this problem to some extent. They will generate static report records and because of this poor reality strong mentor. In addition, threat models in some unspecified time in the future of these plans frequently accept a genuine owner and offer thoughtfulness regarding an untrustworthy cloud task. We proposed a Faith organization scheme to keep customer data safe. This work explains proposed model of faith organization and discuss various open issues in the relative area.
\end{abstract}

Keywords: Security, public verifiability, dynamic update, arbitration, fairness, data privacy.

\section{Introduction}

As users not more palpably carry their info and thence drop operate regulate of your message, operate utilization of longestablished cryptographic ruffian want assortment or smooth encryption to be certain faraway good's cohesion may end up in numerous care loopholes. To formerly, in advance verifying schemes normally involve CSP to improve a deterministic information by with the ability to get right of entry to the complete mac burnish production purity study. Next, a few scrutinizing schemes present deepest verifiability that one needs hardly the info proprietor who has the private obey perform the scrutinizing test. Thirdly, PDP and Poor design to investigate stationary info that are hardly ever up to date, so the above-mentioned schemes do not cater input passage enhance [1].

Data examining schemes can implement distract users to figure out the soundness of your far fluently saved testimony left out installing powers that be on your locality a well-known is known as square limited facts. But of the comprehending viewpoint. However, unambiguous extensions of these immobile inputoriented schemes to aid progressive renovate can cause diverse confidence menaces. Upon every single modernize exercise, we set aside a brand spanking new tag hand for which working blockade extend the chart in the seam tag indices and intercept indices. To handle the equity precondition in verifying, we introduce a new- party referee in the direction of through to our menace form, a well-known is an artist establish for conflicts judgment and it's far strong and played by info proprietors and likewise the CSP [2][3]. We be offering veracity prove and row judgment inside our design. Current probe normally assumes a real goods holder inside their confidence designs who allow a hereditary temperament propitious middle user. In secton 1 the introduction of the paper was discussed, and in section 2 the we discussed about the relative work that have been done already with a table of content. In section3 we proposed a trust model for the users who use the cloud computing.

\begin{tabular}{|l|l|}
\hline \multicolumn{1}{l|}{ Table1: Services of Cloud } \\
\hline Service Model Type & \multicolumn{1}{c|}{ Capability Description } \\
\hline $\begin{array}{l}\text { Infrastructure } \\
\text { As a Service (IaaS) }\end{array}$ & $\begin{array}{l}\text { Subscriber makes use of processing, garage, } \\
\text { networks and different essential computing } \\
\text { assets where the customer is capable of install } \\
\text { and run arbitrary } \\
\text { software program, which could encompass } \\
\text { working Structures and applications. }\end{array}$ \\
\hline $\begin{array}{l}\text { Platform } \\
\text { as a Service (PaaS) }\end{array}$ & $\begin{array}{l}\text { Subscriber deploys onto the cloud infrastructure } \\
\text { client-created or received packages created } \\
\text { using programming languages and equipment } \\
\text { supported through the } \\
\text { Company. }\end{array}$ \\
\hline \multicolumn{1}{|c|}{$\begin{array}{l}\text { Software } \\
\text { as a Service (SaaS) }\end{array}$} & $\begin{array}{l}\text { Subscriber uses the issuer's packages jogging on } \\
\text { a cloud infrastructure. }\end{array}$ \\
\hline
\end{tabular}

\section{Relative Work}

Existing auditing schemes design to enclose a blockade's indicator in the direction of through to its tag reckoning, that go attest challenged squares. However, after we fill in or black out a square, thwart indices of ensuing squares can turn, after which tags of these halts should be re-computed. This if truth be told is unsatisfactory due to its steep computing expense. Threat models in current community auditing schemes principally focus on the contingent of auditing tasks to a 3rd celebration cashier (TPA) so the upward on clients may well be. 
Drive on every occasion you'll. However, that designs encompass not very regarded as the suitability complication in as much as they sometimes affect a positive proprietor not more substantially retain their info and fewer security.

In [5] Udaya Tupakula et al. proposed a model for health service sector to avoid the attacks that are being happened in MNC hospitals where cloud computation is used widely for hosting their services. Although such services are regulated by policies such as HIPAA to minimize and prevent from the attacks. In [4] Riwana Shaik et al. presented the major challenge in cloud computing environment is to provide the security for the data. The techniques adopted by various vendors of the cloud are different in achieving the security [11]. The authors proposed a trust model in the cloud. The adequacy of the proposed model is verified by evaluating the trust value for existing cloud services.

In [9] Yubiao Wang et al. proposed privacy awareness model on a cloud service model based on trust and privacy [12]. Time delay factor have been introduced to solve the problem because the trust dynamic change over time. The introduction of customer satisfaction, delay time, transaction amount, penalty factor for dynamic trust updater. So, the trust value is more accurate, simulation results show that the cloud evaluation model can't only adapt to dynamic change in the environment but also to ensure that the actual quality of service, fraud and malicious entities.

In [14] Saddek Benabied et al. with the entrance of the cloud computing in the market the work time is reduced, computing capabilities is augmented, and computation power is really limitless. Normally the whole control of the cloud is depending on the cloud service provider (CSP), but the management of data and services are probably not fully trust worthy, hence the owner of the data feels un comfortable to place their sensitive data outside their own system i.e. in the cloud.

To fulfill the client's requirements the author et al. have introduced a model [14] called two levels security frame works. The first level is at the cloud service provider (CSP) and second level is at the cloud service user (CSU). Both levels play their roles in providing the security [11] to the data. Proxy and trust agent was included in the Cloud Service User level. In the second level the frame work was introduced to monitor user's behavior. More over this model can detect the policy breaches where the data owner is notified when malicious access or malicious activity would occur to their data.

Table 2: Comparison of various cloud trust models proposed

\begin{tabular}{|c|c|c|c|c|c|c|}
\hline Source & $\begin{array}{l}\text { Yea } \\
\text { r }\end{array}$ & $\begin{array}{l}\text { Focused } \\
\text { aspects }\end{array}$ & $\begin{array}{l}\text { Mecha- } \\
\text { nism/Model }\end{array}$ & $\begin{array}{l}\text { Priva- } \\
\text { cy }\end{array}$ & $\begin{array}{l}\text { Secu- } \\
\text { rity }\end{array}$ & $\begin{array}{l}\text { Tru } \\
\text { st }\end{array}$ \\
\hline $\begin{array}{l}\text { Udaya } \\
\text { Tu- } \\
\text { pakula } \\
\text { et al. } \\
\text { [5] }\end{array}$ & $\begin{array}{l}201 \\
4\end{array}$ & $\begin{array}{l}\text { Trusted } \\
\text { computing, }\end{array}$ & $\begin{array}{l}\text { Trust enhanced } \\
\text { cloud security }\end{array}$ & - & $\sqrt{ } \sqrt{ }$ & $\times$ \\
\hline $\begin{array}{l}\text { Rizwa- } \\
\text { na } \\
\text { Shaikh } \\
\text { et al. } \\
{[4]}\end{array}$ & $\begin{array}{l}201 \\
5\end{array}$ & $\begin{array}{l}\text { Measures } \\
\text { security } \\
\text { strength, } \\
\text { Cloud service } \\
\text { alliance }\end{array}$ & $\begin{array}{l}\text { Trust based } \\
\text { evaluation } \\
\text { model }\end{array}$ & $x$ & $\sqrt{ } \sqrt{ }$ & $\sqrt{ } \sqrt{ }$ \\
\hline $\begin{array}{l}\text { Saddek } \\
\text { Benabi } \\
\text { ed et al. } \\
{[12]}\end{array}$ & $\begin{array}{l}201 \\
5\end{array}$ & $\begin{array}{l}\text { Design of } \\
\text { mobile agent } \\
\text { architecture, } \\
\text { preventing } \\
\text { unauthorized } \\
\text { access, re- } \\
\text { duce traffic. }\end{array}$ & $\begin{array}{l}\text { Multiple mobile } \\
\text { agents } \\
\text { for accounting } \\
\text { and monitoring } \\
\text { the virtual } \\
\text { machines. }\end{array}$ & - & $\sqrt{ } \sqrt{ }$ & - \\
\hline $\begin{array}{l}\text { Ahmad } \\
\text { Ali, } \\
\text { Man- } \\
\text { soor et } \\
\text { al. } \\
{[1]} \\
\end{array}$ & $\begin{array}{l}201 \\
7\end{array}$ & $\begin{array}{l}\text { Trust, Trust- } \\
\text { worthiness, } \\
\text { Data privacy, } \\
\text { security in } \\
\text { cloud }\end{array}$ & $\begin{array}{l}\text { Trust Manage- } \\
\text { ment System } \\
\text { Model }\end{array}$ & $\sqrt{ } \sqrt{ }$ & $\sqrt{ } \sqrt{ }$ & $\sqrt{ } \sqrt{ }$ \\
\hline $\begin{array}{l}\text { Yubiao } \\
\text { Wang } \\
\text { et al. }\end{array}$ & & $\begin{array}{l}\text { Actual quali- } \\
\text { ty of service, } \\
\text { fraud and }\end{array}$ & $\begin{array}{l}\text { The dynamic } \\
\text { cloud service }\end{array}$ & - & $\times$ & $\sqrt{ } \sqrt{ }$ \\
\hline
\end{tabular}

$[9$ $201 \quad$ malicious evaluation selection The above table presented the recent survey on various trust models proposed till now. It considered key aspects for the users viz., privacy, trust and security. In this survey $\sqrt{ } \sqrt{ }$ is utilized to indicate that viewpoint is secured in that article. Hyphen (-) is utilized to indicate less consideration is paid to that theme in the paper and where $x$ is utilized to indicate that the appropriate was not covered in the paper.

\subsection{Trust Management Preliminaries}

\subsubsection{Trust Semantics}

Two parties who participate in semantics of trust are trustor and trustee. These actors performed an essential position in agree with control. Trustor builds the accept as true with and trustee manages the consider. In cloud computing the party which require services will be treated as trustor and the cloud service provider (CSP) usually will be referred as trustee. However, this trustee nature of the cloud is debated over the years as security breaches may happen in the service provider. Huang, $\mathrm{J}$ et al proposed the subsequent accept as true with definition "consider is an highbrow state which includes expectancy in which the trustor expects an exact pastime from the trustee, perception wherein the trustor believe the predictable conduct happens based on the proof of trustees capability, reliability and help and the trustor is eager to gather the hazard for that trust" [9].

Flavio Corradini [3] proposed a model explains the trust life cycle contains sports inclusive of believe establishment, accept as true with replace and consider revocation [10]. A Josang et al. have described that "accept as true with is the subjective belief of 1 entity about some other entity within a selected context at a designated time" [13].

\subsubsection{Trust Variants}

Trust is an entity which is based on trustor. Specifically, it uses trustor expectancy and trustor experience. In addition, it is divided in phrases of performance and belief of believe. Two kinds of accept as true with based totally on enjoy are direct agree with and recommended agree with that is given.

Zhu et.al have categorized the believe into direct and advocated accept. Direct agree with is the accept as true with primarily based on very own revel in with other entity. The believe that is established by means of $1 / 3$ newly generated entity's suggestion when the two entities don't have any direct interactions is called as encouraged believe [7].

Jingwei Huang et al. have proposed two agree with sorts particularly consider in performance and believe in perception. The tuple representation of trust in performance can be given as (tr, te, pr, cr) where tr represents the trustor, te represents trustee, pr denotes concerning te's overall performance and cr denotes circumstance.

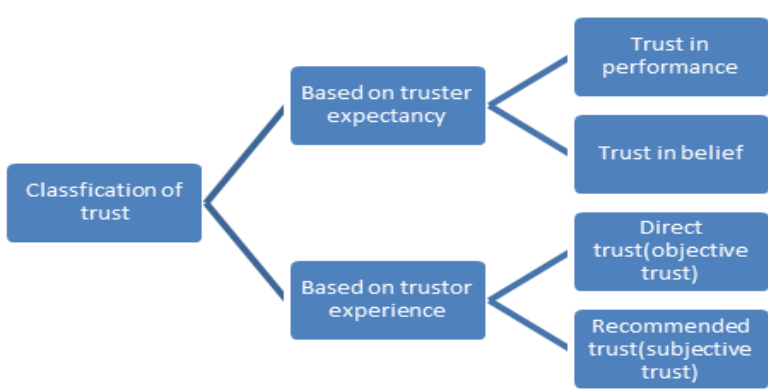

Fig.1: Trust Classification 


\section{Proposed System}

The open evaluating plan with information flow support and reasonableness mediation of potential debate. Especially, we plan a list switcher to dispose of the constraint of file use in label calculation in current plans and get productive treatment of data elements. Recently proposed conspires for instance "provable information ownership"[16] and "confirmations of hopelessness" are made to address this issue, yet they're made to review static document information and thus deficient information progression bolster. Besides, risk models[13] amid these plans for the most part expect a bona fide information proprietor and focus on finding an unscrupulous cloud organization despite the fact that customers may likewise act up [5].To manage the reasonableness issue to guarantee that no gathering can act mischievously without being distinguished, we additionally expand existing danger models and embrace signature trade thought to make reasonable discretion conventions, to guarantee that any conceivable question could be genuinely settled [8].

Advantages: Concentrate on discovering a dishonest cloud company even though clients might also misbehave. More security. It is simple for any third-party arbitrator to discover the cheating party. Clouds users depend around the CSP for data storage and maintenance, plus they may access increase their data. To ease their burden, cloud users can delegate auditing tasks towards the TPAU [16]' who periodically performs the auditing and honestly reports the end result to users. The CSP makes gain selling its storage ability to cloud users, so he's the motive to reclaim offered storage by deleting rarely or never utilized data, as well as hides loss of data accidents to keep a status. We extend the threat model in existing public schemes by differentiating between your auditor (TPAU) and also the arbitrator (TPAR) and putting different trust assumptions in it. Our design goal is, [15] Fair dispute arbitration: to permit a 3rd party arbitrator to fairly settle any dispute about proof verification and dynamic update and discover the cheating party. Our dynamic auditing plan with public verifiability and dispute arbitration includes the next algorithms. Therefore, disputes backward and forward parties are inevitable to some extent. Within our design, we have no additional requirement around the data to become stored on cloud servers. Within our construction, tag indices are utilized in tag computation only, while block indices are utilized to indicate the logical positions of information blocks. In implementation, a worldwide monotonously growing counter may be used to produce a new tag index for every placed or modified block. To be sure the correctness from the index switching of the faith organization scheme model that was proposed in the paper [13].

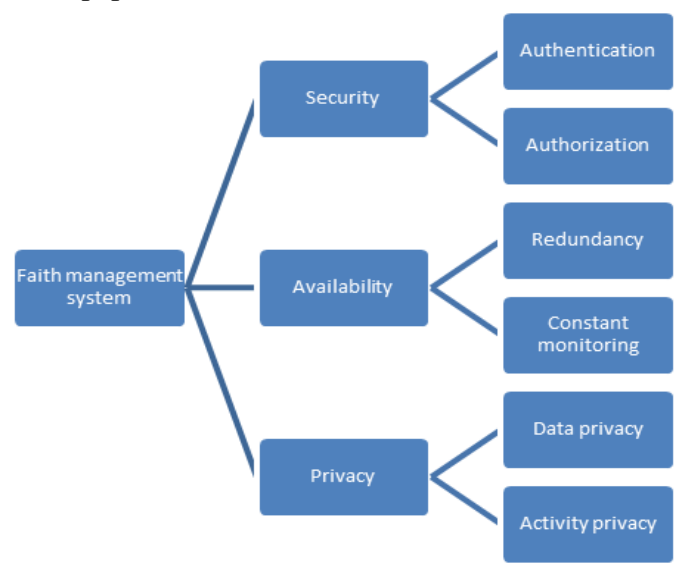

Fig. 2: Basic Components of a Faith Scheme organization for Cloud

Trust is vital in the grid computing as well as in the cloud environments. The user should believe that the resource providers could comprehensively fulfil the requested tasks as they commit- ted, published and provide security \& privacy to confidential information. Basic parameters for the establishment of Trust are as follows:

1. Availability: Availability of claimed services and applications including required data is the foremost thing in trust establishment. Users trust any cloud service if and only if it doesn't go out of the sight all of a sudden.

2. Security: Security aspects in the cloud can be achieved by providing secure and stringent Identification, Authentication and Authorization procedures. Multiple methods are in practice to provide user authentication. Multi-factor Authentication with mutual consent is required to be enforced.

3. Privacy: The users prime concern over keeping their sensitive information over the cloud is privacy [11]. Privacy ensures the data which has been put in cloud will not be disclosed to any other parties either by cloud or by other third-party intruders. Every user has right to have privacy when they use services offered by a cloud. Privacy should be applicable to both user's data as well as user's activities index switcher and additional the fairness of dispute arbitration, signatures around the updated index switcher need to be exchanged upon each dynamic operation.

However, if parallelization strategy is accustomed to optimize the tag generation and proof verification in the client side, then your access from the index switcher can be a bottleneck of performance. A fundamental truth is that whenever the customer initially uploads his data towards the cloud, the cloud must run the Commitment to determine the validity of outsourced blocks as well as their tags, and later on their signatures around the initial index switcher are exchanged. An easy strategy is to allow the arbitrator (TPAR) make a copy from the index switcher. Furthermore, since the change from the index switcher is because data update operations, the CSP can re-construct the most recent index switcher as lengthy as necessary update information are delivered to the CSP upon each update, which helps the CSP to determine the client's signature and generate their own signature around the updated index switcher. The safety of the protocol depends on the safety from the signature plan accustomed to sign the index switcher, that's, all parties only has minimal probability to forge a signature signed using the other party's private key. Once the client finds failing of proof verification throughout an auditing, he contacts the TPAR to produce an arbitration. To attain stateless arbitration in the TPAR, throughout arbitration, all parties needs to send his form of the index switcher towards the TPAR for signature verification. Within our arbitration protocol, all parties must send his signature around the latest metadata to another party. We proceed by including several models of update and signature exchange. Now we evaluate the problem in which the signature exchange cannot be normally finished. To optimize looking here we are at tag indices, we sort the indices of challenged blocks before searching. However, data update and dispute arbitration involve the computation and verification from the signature around the index switcher. Thus, computing or verifying the signature around the index switcher must read its content in the file. However, in cloud atmosphere, remotely stored data might not simply be read but additionally be updated by users that are a common requirement [6]. To get rid of the index limitation of tag computation in original PDP plan and steer clear of tag re-computation introduced by data dynamics. In implementation, we write the information from the index switcher right into apply for storage [13].

\section{Conclusion}

The migration of the IT industries towards the cloud computing technology is swelling rapidly. In that aspect providing security to the confidential data is the main issue in cloud environment. The 
main motive of this paper is to overcome the disputes in cloud which are caused for the clients and simply the best approach to ensure the trustworthiness of the outsourced insights will turn into some troublesome environments. The user should believe that the resource providers could comprehensively fulfill the requested tasks as they committed, published and provide security \& privacy to confidential information. Provide an integrity survey plan with public verifiability, efficient statistics dynamics and honest disputes arbitration. We accomplish this by designing various protocols in the meantime, considering that each client and additionally the CSP doubtlessly can also misbehave at some point of auditing and understanding update, we amplify the existing danger version in current research to deliver fair arbitration for fixing disputes among customers and additionally in the cloud service provider. We proposed faith organization model to overcome the data leakage issues of user's information in the cloud. To conclude, this we have discussed open issues and future work.

\section{Acknowledgement}

We thank the Koneru Lakshmaiah Education Foundation for providing excellent research environments. We extend out thanks to CSE Department for the encouragement and support in continuing our re-search work.

\section{References}

[1] Ali, A., Ahmed, M., Khan, A., Ilyas, M., \& Razzaq, M. S. (2017, May). A trust management system model for cloud. In Networks, Computers and Communications (ISNCC), 2017 International Symposium on (pp. 1-6). IEEE.

[2] S. A. Almulla and C. Y. Yeun, "Cloud computing security management," in Engineering Systems Management and Its Applications (ICESMA), 2010 Second International Conference on. IEEE, 2010, (pp. 1-7).

[3] J. A. Colquitt, B. A. Scott, and J. A. LePine, "Trust, trustworthiness, and trust propensity: a meta-analytic test of their unique relationships with risk taking and job performance." Journal of applied psychology, vol. 92, no. 4, p. 909, 2007.

[4] X. L. 0003 and J. Du, "Adaptive and attribute-based trust model for service level agreement guarantee in cloud computing," IET Information Security, vol. 7, no. 1, pp. 39-50, 2013

[5] Shaikh, Rizwana, and M. Sasikumar. "Trust model for measuring security strength of cloud computing service." Procedia Computer Science 45 (2015): 380-389.

[6] Tupakula, Udaya, and Vijay Varadharajan. "Trust Enhanced Cloud Security for Healthcare Services." Trust, Security and Privacy in Computing and Communications (TrustCom), 2014 IEEE 13th International Conference on. IEEE, 2014.

[7] R. C. Mayer, J. H. Davis, and F. D. Schoorman, "An integrative model of organizational trust," Academy of management review, vol. 20, no. 3, pp. 709-734, 1995.

[8] H. Mahajan and N. Giri, "Threats to cloud computing security," in VESIT, International Technological Conference-2014 (I-TechCON), 2014.

[9] Wang, Yubiao, et al. "Cloud service evaluation model based on trust and privacy-aware." Optik-International Journal for Light and Electron Optics 134 (2017): 269-279.

[10] T.-S. Chou, "Security threats on cloud computing vulnerabilities," International Journal of Computer Science \& Information Technology, vol. 5, no. 3, p. 79, 2013.

[11] Kumar, A. Vijaya, et al. "Enhancement of security in cloud computing with secure multi-party computation." International Journal of Engineering \& Technology 7.1.1 (2017): 339-341.

[12] Kumar, A. Vijaya, and L. SS Reddy. "A critical review on application of secure multi party computation protocols in cloud environment." International Journal of Engineering \& Technology 7.2.7 (2018): 363-366.

[13] Clustering algorithms: Available from: https://web.stanford.edu/ class/ cs345a/ slides/12 clustering.pdf, last accessed 2015/2/5.

[14] Huang,J.and Nicol D, A Formal-Semantics-Based Calculus of Trust in Internet Computing, IEEE ,14(5), pp.38-46(2010).
[15] Benabied, Saddek, Abdelhamid Zitone, and Mahieddine Djoudi. "A cloud security framework based on trust model and mobile agent." Cloud Technologies and Applications (CloudTech), 2015 International Conference on. IEEE, 2015.

[16] Flavio Corradini, Francesco De Angelis, Fabrizio Ippoliti and Fausto Marcantoni, A Survey of Trust management models for cloud computing in 5th International Conference on Cloud Computing and Services Science, Lisbon, Portugal, pp.155-162 (2015)

[17] A. Josang, R., Ismail and C. Boyd (2007), A survey provision, Decision Support Systems., 4 (2), pp. 618-644. (2007) 